\title{
Satellite-induced electron acceleration and related auroras
}

\author{
S. L. G. Hess (1), P.A. Delamere (2), B. Bonfond (3), V. Dols (2) \\ (1) LATMOS, Université Versailles-St Quentin, UPMC, IPSL/CNRS, France (sebastien.hess@latmos.ipsl.fr) \\ (2) LASP, University of Colorado, Boulder,USA \\ (3) SwRI, Boulder, USA
}

\begin{abstract}
Satellite-induced auroral emissions are known since decades, in particular those associated with the interaction of Io with the Jovian ionosphere. These emissions range from low frequency radio to UV. Flyby of Io allowed to better understand the power generation close to the satellite, and showed the existence of electron beams accelerated at high latitude. We will present a study of the power transfer between the local interaction at Io and the electron accelerated close to Jupiter. It shows that Alfvén acceleration can explain the morphology and brightness of the Io-related auroraeand the observed accelerated electrons in Io's wake. The study is extended to the Europa, Ganymede and Enceladus for which auroral emissions have been observed, as well as to Callisto and to the principal inner satellites of Saturn.
\end{abstract}

\section{Introduction}

The auroral emissions induced by the Io interaction with the Jovian magnetosphere are observed since decades. These emissions extend over a large spectrum, from low frequency radio to Infrared, visible and UV. The total power emitted reaches more than $10^{10}$ Watts, for the main Io spot only and up to $10^{11}$ Watts for the whole Io footprint. On the other end, the Io local interaction with the Jovian magnetic field delivers about $10^{12}$ Watts. Thus few percents of the power generated at Io have to be transferred to the electrons above the sole main spot.

The most commonly accepted mechanism for transferring the power from Io the the acceleration region, located at high latitude, is the propagation of an Alfvén wave packet. However, this wave packet has to be partially reflected along its way. Moreover, only a small part of the wave packet power is transfered to the electron.
We present here the conditions under which the correct efficiency of the power transfer from Alfvén wave to electrons are reached, and show that filamentation of the Alfvén wing - that is, of the current system - is necessary.

\section{Known interactions}

Io, Europa, Ganymede and Enceladus are known to generate auroral emissions on top of their respective parent planet ionosphere. The power needed to produce the observed emissions can be determined, and the power generated at the satellite estimated. Hence, we can estimate the actual efficiency of the power transfer. We then compute theoretical power transfer efficiency under different physically relevant assumptions, and deduce that filamentation of the Alfvén wave is needed.

\section{Other satellites}

We extend our study to other satellite which could potentially generate observable auroral emissions. The values we predict are discussed, some of them being in support of claimed observations (e.g. Callisto footprint).

\section{Modulation of the interactions}

We also present a study of the modulation of the Io footprint brightness with Io's longitude, and discuss it in regards of our model.

\section{Acknowledgements}

SH would like to thank the conveners for inviting him, and D. Swift, F. Mottez, F. Bagenal and L.C. Ray for their respective support and/or participation to the studies presented here. 